Open Access

\title{
Lesion Location in Clinical Significance of Incidental Colorectal FDG Uptake
}

To the Editor:

We agree with Roh et al. ${ }^{1}$ that cancerous and pre-cancerous lesions may be harboured by the finding of incidental F18-fluorodeoxyglucose (FDG) uptake on positron emission tomography (PET) scan. This study-and our own data ${ }^{2}$-advocates for urgent investigation of such findings, especially considering the treatment implications for patients undergoing therapy for other established malignancies (which was the principal indication for PET).

With regard to measurement of maximum standardised uptake values (SUVmax)-favoured to a greater extent in the accompanying editorial commentary ${ }^{3}$-we found in our study (and in our clinical work in general) that it was not adequately useful in assessing malignancy likelihood. We, too, found the degree of overlap in the range of values was often confounding. While utilising imaging clues to predict malignancy potential is very useful, SUVmax seemed not to be as helpful as we initially hoped.

In our experience, focal FDG uptake is much more concerning for significant pathology than segmental or diffuse FDG uptake, regardless of the SUVmax value. We also found that anatomical location (with lesions classified as simply as being in the "proximal" or "distal" colon) had a higher predictive value for malignancy. It is also important to note that other studies of this phenomenon have found that as many as $17 \%{ }^{4}$ to $32 \%{ }^{5}$ of foci of abnormal colonic FDG uptake represented aetiologies other than malignancy.

Indeed, recent publications suggest that some of this uptake may be related to bacterial labelling in the colonic lumen. ${ }^{6}$ More work is required to elucidate the pathophysiology and possible causes of FDG uptake in the colon. Regardless, based on study of Roh et al. ${ }^{1}$ and our own, ${ }^{2}$ consideration should al- ways be given to investigating focally increased colonic FDG uptake to exclude neoplasia. Additional clues in establishing the clinical significance are also potentially very helpful.

\section{Conflicts of Interest}

The authors have no financial conflicts of interest.

Joseph C. Lee ${ }^{1,2}$, Gemma F. Hartnett ${ }^{3}$ and Aravind S. Ravi Kumar ${ }^{2,4}$

${ }^{1}$ Department of Nuclear Medicine, The Prince Charles Hospital, Chermside, ${ }^{2}$ University of Queensland School of Medicine, Herston, ${ }^{3}$ Department of Medical Oncology,

Redcliffe General Hospital, Redcliffe, ${ }^{4}$ Department of Nuclear Medicine and Queensland PET Service, Royal Brisbane and Women's Hospital, Herston, Australia

\section{REFERENCES}

1. Roh SH, Jung SA, Kim SE, et al. The clinical meaning of benign colon uptake in 18F-FDG PET: comparison with colonoscopic findings. Clin Endosc 2012;45:145-150.

2. Lee JC, Hartnett GF, Hughes BG, Ravi Kumar AS. The segmental distribution and clinical significance of colorectal fluorodeoxyglucose uptake incidentally detected on PET-CT. Nucl Med Commun 2009;30: 333-337.

3. Jang BI. Benign colonic 18F-FDG uptake on whole-body FDG-PET scan. Clin Endosc 2012;45:109-110.

4. Israel O, Yefremov N, Bar-Shalom R, et al. PET/CT detection of unexpected gastrointestinal foci of 18F-FDG uptake: incidence, localization patterns, and clinical significance. J Nucl Med 2005;46:758-762.

5. Kamel EM, Thumshirn M, Truninger K, et al. Significance of incidental 18F-FDG accumulations in the gastrointestinal tract in PET/CT: correlation with endoscopic and histopathologic results. J Nucl Med 2004; 45:1804-1810

6. Wang K, Chen YC, Palmer MR, et al. Focal physiologic fluorodeoxyglucose activity in the gastrointestinal tract is located within the colonic lumen. Nucl Med Commun 2012;33:641-647. 


\section{Response:}

To the Editor:

The exact interpretation of clinical meaning of benign colon uptake in ${ }^{18} \mathrm{~F}$-fluorodeoxyglucose positron emission tomography (FDG-PET) is not easy because the result can be influenced by diverse causes from even physiologic lesion to malignant lesion. In considering the clues to determine the clinical meaning of benign colon FDG uptake, we fully agree with Lee et al's opinion that maximum standardized uptake values (SUVmax) only was not adequately useful in assessing malignancy and focal FDG uptake was much more concerning for significant pathology than segmental or diffuse FDG uptake, regardless of the SUVmax value.

Although many studies have showed that mean maximal SUVmax was higher in malignant lesion than benign lesion or normal tissue, ${ }^{1-5}$ increased mean SUVmax was usually observed in patients with cancer as well as other patients with polyps or even normal patients, with broad overlap between groups. Some studies ridiculously reported that there were no significant difference in SUVmax among the malignant, premalignant and normal lesion. ${ }^{6-10}$ Therefore, SUVmax alone seemed to be not adequately useful in assessing malignancy as Lee et al.s opinion.

The role of anatomical location or size in determining meaning of colonic FDG uptake is controversial. Lee et al. ${ }^{11}$ reported that the positive predictive value for malignant or premalignant pathology was higher in the proximal colon than in the distal colon. However, Peng et al. ${ }^{1}$ reported that FDG uptake in the right colon showed a higher false positive rate, than in the distal colon and rectum $(66.2 \%$ vs. $36.7 \%, p=0.004)$ and FDG uptake in the right colon was a negative predictive factor for finding cancer or polyps. Further studies with large number of patients are necessary to clarify the conflicting results of these two studies.

The size of the FDG uptake lesion was another considering factor. Peng et al. ${ }^{1}$ reported the size between cancer group and polyps group was significantly different $(4.3 \mathrm{~cm}$ vs. $1.4 \mathrm{~cm}$, $p=0.009$ ) and tumor size was not related to the SUVmax value. However, there was no significant correlation between the size of lesions and pathology in our study. ${ }^{12}$

The uptake pattern, focal versus diffuse or segmental, is the most considering factor in determining the nature of the lesion with FDG uptake. We recently showed that positive predictive value of benign focal colon FDG uptake was higher than them of diffuse one. ${ }^{12}$ This result is in line with that of
Lee et al.

Taken together, the patients showing benign colonic FDG uptake should be further evaluated by colonoscopy, especially in the cases with focal uptake pattern. The value of SUVmax should be cautiously considered because of broad overlap in different lesions. The role of location and size of the lesion should necessitate further evaluation in large cohort.

\section{Conflicts of Interest}

The authors have no financial conflicts of interest.

\section{Sun Hee Roh and Sung-Ae Jung}

Department of Internal Medicine, Ewha Medical Reseach Institute and Ewha Womans University School of Medicine, Seoul, Korea

\section{REFERENCES}

1. Peng J, He Y, Xu J, Sheng J, Cai S, Zhang Z. Detection of incidental colorectal tumours with 18F-labelled 2-fluoro-2-deoxyglucose positron emission tomography/computed tomography scans: results of a prospective study. Colorectal Dis 2011;13:e374-e378.

2. Strauss LG, Klippel S, Pan L, Schönleben K, Haberkorn U, Dimitrakopoulou-Strauss A. Assessment of quantitative FDG PET data in primary colorectal tumours: which parameters are important with respect to tumour detection? Eur J Nucl Med Mol Imaging 2007;34:868-877.

3. Keogan MT, Lowe VJ, Baker ME, McDermott VG, Lyerly HK, Coleman RE. Local recurrence of rectal cancer: evaluation with F-18 fluorodeoxyglucose PET imaging. Abdom Imaging 1997;22:332-337.

4. Weston BR, Iyer RB, Qiao W, Lee JH, Bresalier RS, Ross WA. Ability of integrated positron emission and computed tomography to detect significant colonic pathology: the experience of a tertiary cancer center. Cancer 2010;116:1454-1461.

5. Chen YK, Kao CH, Liao AC, Shen YY, Su CT. Colorectal cancer screening in asymptomatic adults: the role of FDG PET scan. Anticancer Res 2003;23:4357-4361.

6. Treglia G, Calcagni ML, Rufini V, et al. Clinical significance of incidental focal colorectal (18)F-fluorodeoxyglucose uptake: our experience and a review of the literature. Colorectal Dis 2012;14:174-180.

7. Isobe K, Hata Y, Sakaguchi S, et al. The role of positron emission tomography in the detection of incidental gastrointestinal tract lesions in patients examined for lung cancer. Nihon Kokyuki Gakkai Zasshi 2010; 48:482-487.

8. Pandit-Taskar N, Schöder H, Gonen M, Larson SM, Yeung HW. Clinical significance of unexplained abnormal focal FDG uptake in the abdomen during whole-body PET. AJR Am J Roentgenol 2004;183:1143-1147.

9. Kei PL, Vikram R, Yeung HW, Stroehlein JR, Macapinlac HA. Incidental finding of focal FDG uptake in the bowel during PET/CT: CT features and correlation with histopathologic results. AJR Am J Roentgenol 2010; 194:W401-W406.

10. Israel O, Yefremov N, Bar-Shalom R, et al. PET/CT detection of unexpected gastrointestinal foci of 18F-FDG uptake: incidence, localization patterns, and clinical significance. J Nucl Med 2005;46:758-762.

11. Lee JC, Hartnett GF, Hughes BG, Ravi Kumar AS. The segmental distribution and clinical significance of colorectal fluorodeoxyglucose uptake incidentally detected on PET-CT. Nucl Med Commun 2009;30: 333-337.

12. Roh SH, Jung SA, Kim SE, et al. The clinical meaning of benign colon uptake in 18F-FDG PET: comparison with colonoscopic findings. Clin Endosc 2012;45:145-150. 